\title{
Hybrid mapping in VLBI and spurious symmetrization
}

\author{
M. Massi ${ }^{1,2}$ and S. Aaron ${ }^{1,2}$ \\ 1 Max-Planck-Institut für Radioastronomie, Auf dem Hügel 69, D-53121 Bonn, Germany \\ 2 Joint Institute for VLBI in Europe, Postbus 2, 7990 AA, Dwingeloo, The Netherlands
}

Received June 29; accepted December 9, 1998

\begin{abstract}
The standard procedure used in hybrid mapping of VLBI data is examined. That this procedure can converge on wrong solutions has been pointed in the past by many authors. Walker (1996) indicated the bias in the resultant data due to the use of a point source as starting model. Bååth (1989) suggested the use of the original data set in each iteration of self calibration, and not the use of the corrected one created by the previous iteration. Linfield (1986) analyzed the role of the $u-v$ coverage and lack of intermediate spacings. However, the full procedure needed to avoid false features is at present not clear and only experience with imaging helps the user to avoid them. In this paper we demonstrate that the problem is connected with the non-linear nature of self calibration which leaves initial wrong assumptions frozen in the final solution. We demonstrate how large closure phase values linearize the process and we show a simple modification of the standard procedure which helps to avoid creation of false features in the imaging.
\end{abstract}

Key words: methods: analytical - methods: data analysis - techniques: interferometric instrumentation: interfereometers

\section{Introduction}

When one telescope of a VLBI network is very displaced from the others, a large hole results in the $u-v$ coverage. An example is in observations where one telescope is on a satellite: the baselines between two ground stations will be much shorter than those between a ground station and the satellite. The hole in the $u-v$ coverage appears between the baselines connecting pairs of ground based stations and those much longer connecting each ground station with the orbiting telescope. Such a hole may also occur in observations using EVN telescopes in China and Europe or

Send offprint requests to: M. Massi using both EVN and VLBA telescopes. By studying simulations of orbiting VLBI projects, Linfield (1986) showed that the hybrid mapping procedure usually used to self calibrate ground-based VLBI observations created artificial structures when significant holes were present in the $u-v$ data. These structures tended to be symmetric with real structure and were not removed by the standard processing despite many iterations of the process.

The standard hybrid mapping scheme is sketched in Fig. 1. Some initial model for the source is chosen, typically a point source. Model visibilities are derived and self calibration determines antenna based corrections to the $u-v$ data by minimizing the expression

$$
L=\sum_{i \neq k} w_{i k}\left|A_{i k_{\mathrm{o}}} \mathrm{e}^{\jmath \psi_{i k_{\mathrm{o}}}}-g_{i} g_{k} A_{i k_{\mathrm{m}}} \mathrm{e}^{\jmath\left[\psi_{i k_{\mathrm{m}}}+\left(E_{i}-E_{k}\right)\right]}\right|^{2},
$$

where $w_{i k}$ are the baseline weights, $A_{i k_{\mathrm{o}}}$ and $A_{i k_{\mathrm{m}}}$ are the observed and model visibility amplitudes, $\psi_{i k_{\mathrm{o}}}$ and $\psi_{i k_{\mathrm{m}}}$ are the observed and model visibility phases, $g_{i}$ and $E_{i}$ are the amplitude and phase corrections for antenna $i$. The corrected data are Fourier transformed and a new model is determined by applying the CLEAN algorithm to the resultant image. New model visibilities are generated, and Eq. (1) is again used to determine a new set of corrections. This process is repeated (called Loop "A" in Fig. 1) until convergence to a constant model is achieved.

We will show in Sect. 4 that for small values of the closure phase the self calibration process is non-linear and is most sensitive to the choice of initial model. Therefore, we suggest a modification to the standard hybrid mapping procedure in that the model derived by CLEAN be used as the initial model for the original data, replacing the point source used the first time through the process. We call this Loop "B" in Fig. 1. We show empirically in Sect. 2 that this modified procedure eliminates the spurious structures created by standard hybrid mapping, enabling the calibration to efficiently converge to an acceptable solution. 


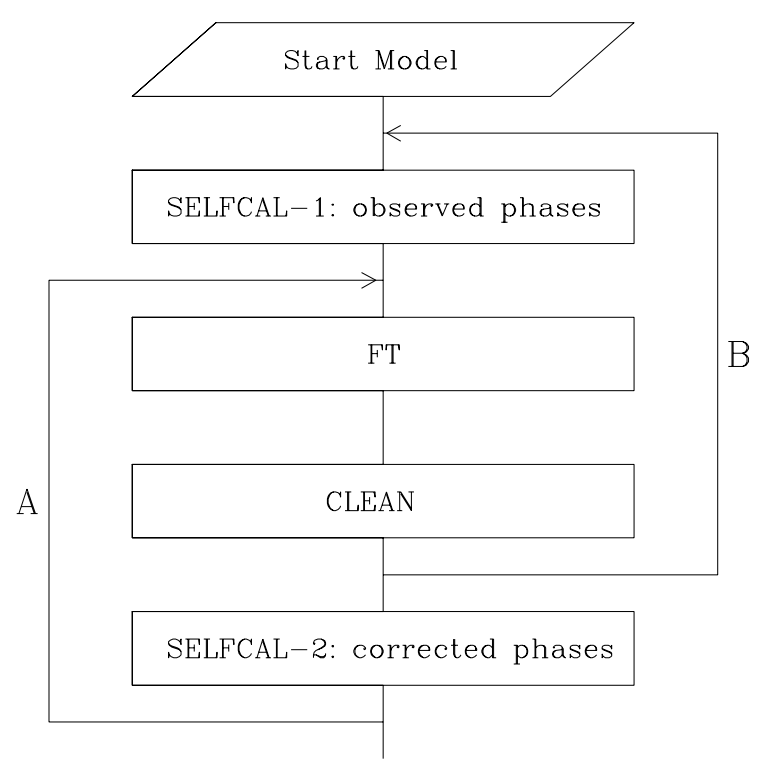

Fig. 1. Hybrid mapping scheme

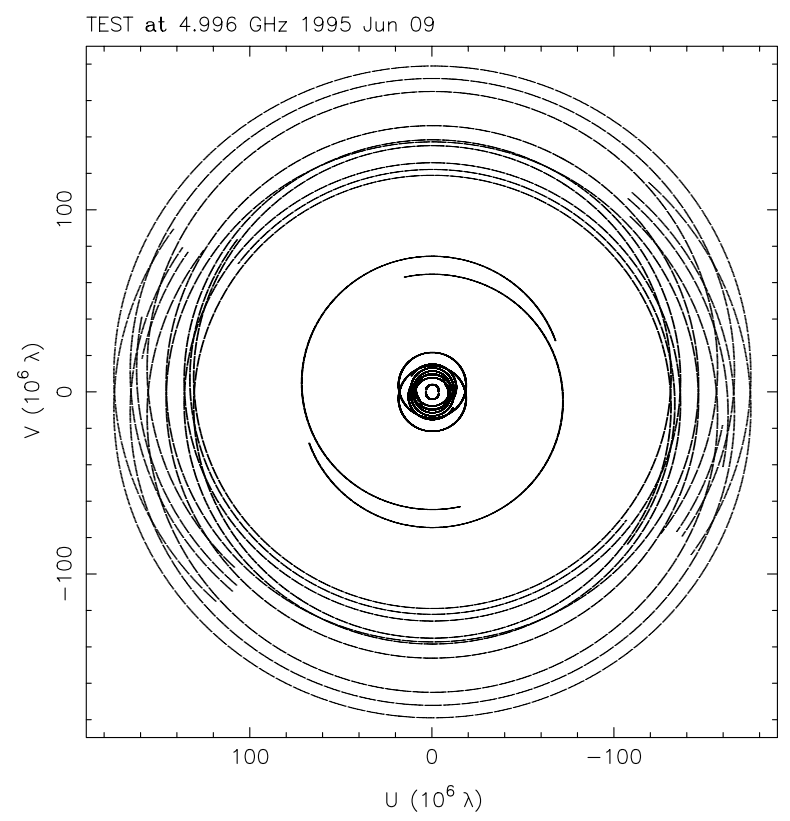

Fig. 2. The $u-v$ coverage of a simulated array consisting of selected EVN and VLBA telescopes

\section{Simulation results}

Using the program FAKE of the Caltech VLBI package, we simulated an observation of a source with a simple corejet morphology using the $u-v$ coverage shown in Fig. 2 . This coverage is generated by an array of selected EVN stations and two VLBA telescopes (MK and KP). The presence of the latter two telescopes provides, together with long baselines to the western EVN telescopes, one baseline at intermediate spacings in the $u-v$ plane.

We introduced random, antenna-based phase errors into the $u-v$ data, which will be removed by the hybrid mapping sequence. The aim of our tests will be, in

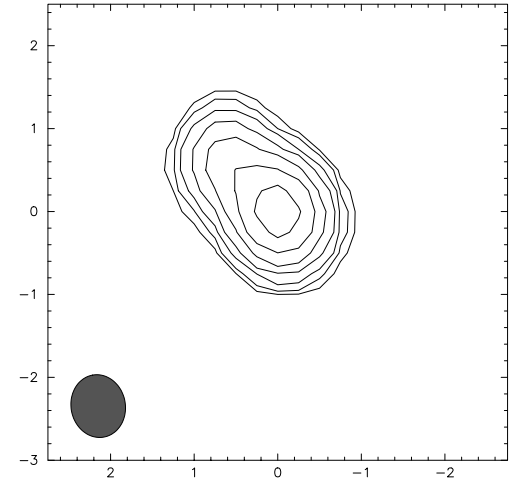

Fig. 3. Map for comparison with the successive tests. The image has been produced by using the true model as input model

case of two slightly different $u v$ coverages, to try to reproduce the map of Fig. 3 (obtained by using the true model as input model).

First, we processed the corrupted data using the standard procedure described in Sect. 1, i.e., repeated iterations of self calibration using Loop "A" with a point source as an initial model. We will refer to this as Test-1 below. The result is shown in Fig. 4. As one can see the hybrid mapping sequence is able in this case to reproduce the map of Fig. 3. No artificial features have been added.

Next, we deleted the single intermediate spacing baseline from the $u-v$ data, and again processed the data with the standard procedure. We will refer to this as Test-2A. We find the image shown in Fig. 5. Spurious structure now appears in the image, extending southwest of the core. As will be shown in Sect. 4, this effect is entirely due to the lack in the self calibration solution of closure phase values related to those triangles of baselines previously using the now flagged intermediate baseline.

A surprising discovery is that excluding the spurious emission region from the clean model does not remove it from the corrected data. Even in this case, in which one can distinguish the spurious features from the real features, Loop "A" continues to generate spurious emission. After 10 iterations with Loop "A", using CLEAN boxes to exclude the spurious feature, we are still left with traces of artificial symmetrization, as we show in Fig. 6 .

Finally, with the intermediate baseline deleted, we processed the $u-v$ data using our modified procedure (Loop "B"), which we refer to as Test-2B below. The result after 3 iterations is shown in Fig. 7. We again have an image that agrees well with the true structure of the simulated source.

The total calibration at the end of repeated iterations of Loop "A" consists of the sum of the corrections derived in each iteration. That this does not converge to the proper result implies the process is non-linear and sensitive to the choice of the initial model. We will show this analytically in Sect. 4. 


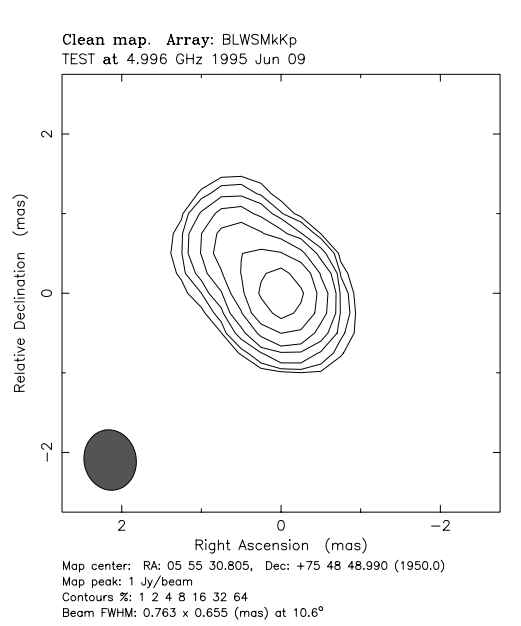

Fig. 4. Test-1: the final image obtained with the full $u-v$ coverage of the sample data set

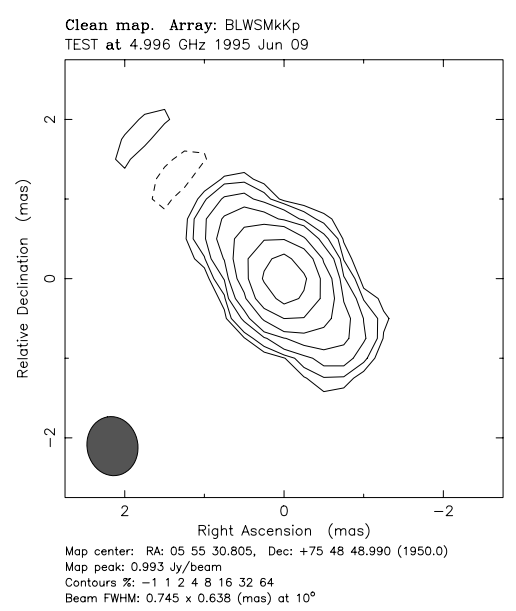

Fig. 5. Test-2A: the final image obtained using the standard self calibration technique on the $u-v$ data from which the lone intermediate length baseline has been deleted

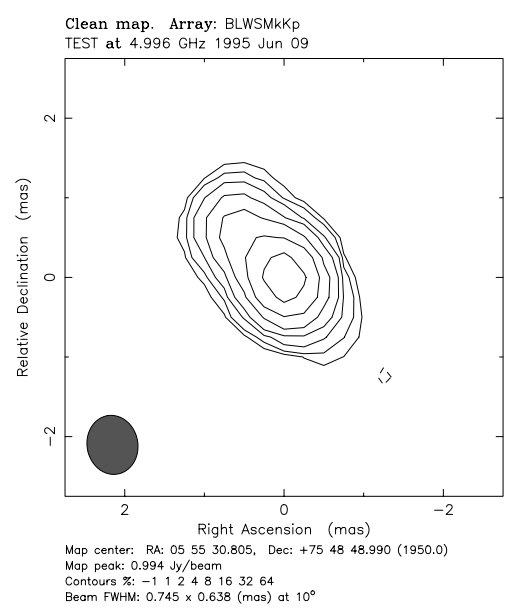

Fig. 6. Same as Fig. 5 but setting (for 10 iterations) CLEAN boxes excluding spurious emission

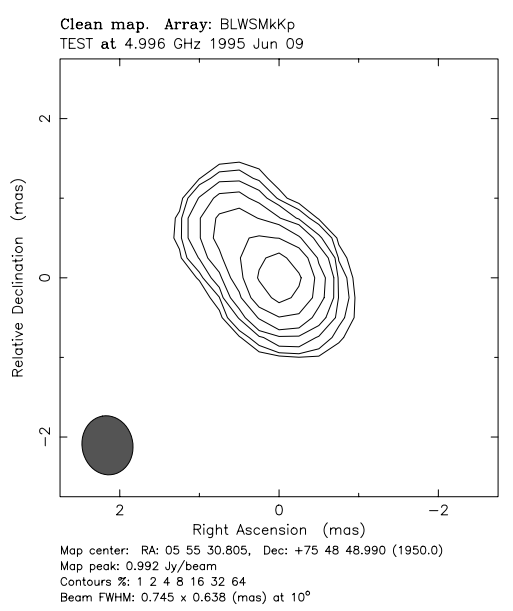

Fig. 7. Test-2B: the final image obtained using our modified self calibration algorithm (see text) on the same $u-v$ data as previously

\section{Closure phase}

The closure phase $\Phi$ is a combination of phases $\psi$ around a triangle of baselines, defined as

$\Phi_{i j k}=\psi_{i j}+\psi_{j k}-\psi_{i k}$.

When a telescope is quite displaced from the other two telescopes (i.e., an unbalanced triangle), the two longest baselines become nearly parallel give essentially identical phases, but with opposite sign. Their contributions therefore almost cancel in the closure equation. The only remaining contribution is that of the shortest baseline; when the source is unresolved on that baseline, the result is a closure phase whose magnitude is only a few degrees.

As shown by Massi (1989), the contribution of the longest baselines to the closure phase is a function of the ratio $s / L$ between the shortest $(s)$ and the longest $(L)$ leg of the closure phase triangle. For a very small ratio (i.e., $s / L \ll 0.3)$ the contribution may drop to a few degrees. This can be seen, for example, in the plots of closure phase shown in Fig. 8. The triangle at the top of the figure makes use of the intermediate length baseline MK to KP (the one flagged in Test-2 of Sect. 2) and has $s / L \simeq 0.3$. The closure phase for this triangle reaches a value of about $40^{\circ}$, significantly larger than the $12^{\circ}$ and $8^{\circ}$ reached for the other two triangles shown, with ratios of $s / L \simeq 0.07$ and 0.05 respectively.

In conclusion in the case of missing intermediate spacing information the observed closure phase $\Phi_{\mathrm{o}}$ is a small quantity. While at first glance, this would imply a quite slow convergence, instead it gives rise, as shown in Sect. 2, to a very serious problem when the adopted starting model is a point source and Loop " $\mathrm{A}$ " is followed. In the next section we show why for cases of uniform $u-v$ coverage Loops "A" and "B" are equivalent, but for cases where only low closure phase values are present (associated with unbalanced triangles), differences become apparent. 


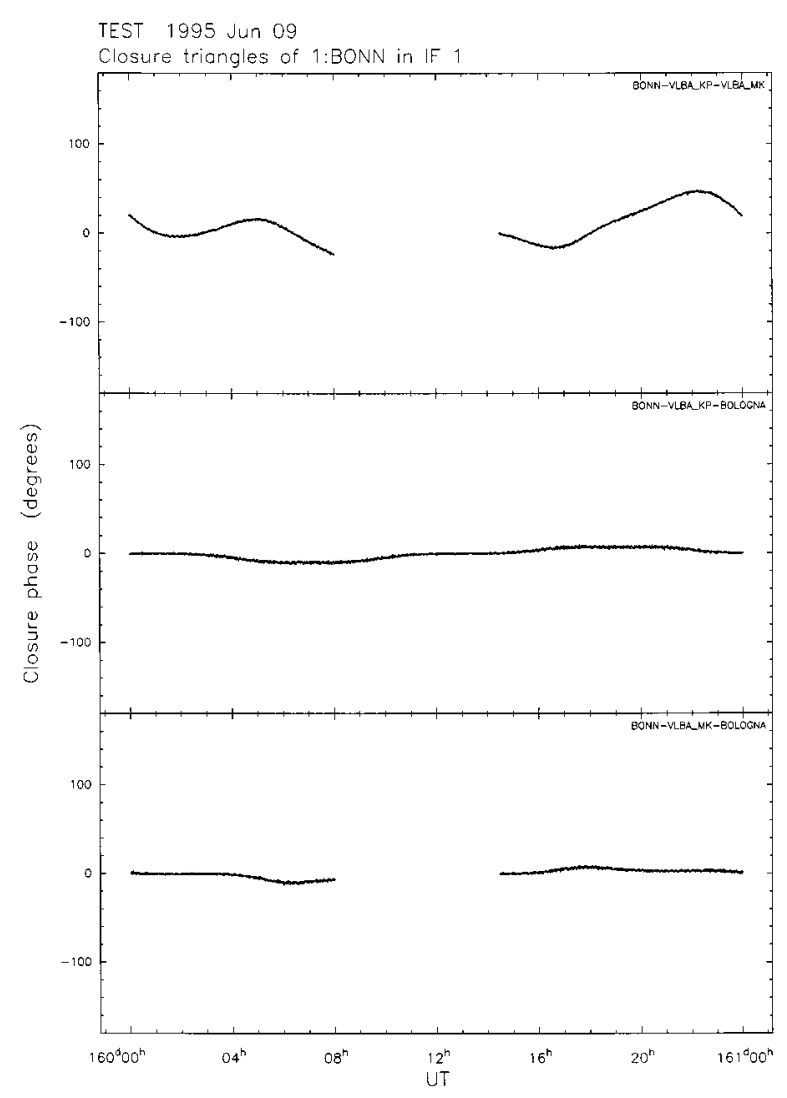

Fig. 8. Observed closure phase for selected triangles

\section{Theory}

In the first part of this section we show how Loop "A" and Loop "B" produce the same results only when the self calibration process is linear. In the second part we discuss the role played by the closure phase in linearizing self calibration.

\subsection{Linear case}

Self calibration, based on the algorithm of Schwab (1980), estimates the errors affecting the observed phase of the visibility function in terms of antenna errors $E$ by minimizing Eq. (1). We may express the observed visibility phase $\psi_{\mathrm{o}}$ on a baseline $i k$ as a combination of the true phase $\psi_{\mathrm{t}}$ and the antenna errors $E$ at the two telescopes as

$\psi_{i k_{\mathrm{o}}}=\psi_{i k_{\mathrm{t}}}+\left(E_{i}-E_{k}\right)$.

We may characterize the difference $\epsilon$ between the true visibility phase $\psi_{\mathrm{t}}$ and the phase $\psi_{\mathrm{m}}$ predicted by a given source model as

$\psi_{i k_{\mathrm{t}}}=\psi_{i k_{\mathrm{m}}}+\epsilon_{i k}$.

By solving for antenna-based errors, the closure phase on a given triangle $i j k$ is preserved, which implies the constraint:

$<E_{i}-E_{j}>+<E_{j}-E_{k}>-<E_{i}-E_{k}>=0$.
We can see that by substituting Eq. (3) and Eq. (4) in Eq. (5), we have:

$$
\begin{gathered}
\left(\psi_{i j_{\mathrm{o}}}-\psi_{i j_{\mathrm{m}}}\right)+\left(\psi_{j k_{\mathrm{o}}}-\psi_{j k_{\mathrm{m}}}\right)-\left(\psi_{i k_{\mathrm{o}}}-\psi_{i k_{\mathrm{m}}}\right)+ \\
-\left(\epsilon_{i j}+\epsilon_{j k}-\epsilon_{i k}\right)=0
\end{gathered}
$$

(where hereafter we omit the baseline subscript for the visibility phases.)

By defining $\Phi_{\mathrm{o}}$ and $\Phi_{\mathrm{m}}$ the observed and the model closure phases (Eq. 2), we have:

$\epsilon_{i j}+\epsilon_{j k}-\epsilon_{i k}=\Phi_{\mathrm{o}}-\Phi_{\mathrm{m}}$.

Where we omit the subscript " $i j k$ " for the closure phases. In other words the estimation of the $\epsilon$ 's depend on the closure phase. How the difference between the observed and model closure phases is distributed among the individual errors $\epsilon$ it depends upon the weights attributed to the various baselines. Since we want here to illustrate in general the role played by the closure phase in the solution, we assume for simplicity equal weights, and estimations of the errors like:

$\epsilon=\epsilon_{i k}=\epsilon_{j k}=-\epsilon_{i j}=-\left(\Phi_{\mathrm{o}}-\Phi_{\mathrm{m}}\right)$.

Now we can see the impact of the closure phase in the self calibration solution. Let us examine the case of a pointlike source as input model. In this case the estimation in Eq. (8) of the error $\epsilon$ depends entirely on the observed closure phase, since in this case $\Phi_{\mathrm{m}}=0$. Let us assume that the observed closure phase is a small quantity, $\Phi_{\mathrm{o}} \simeq 0$, as it is in our simulated $u-v$ data set with the intermediate baseline deleted. The error estimation then becomes a small quantity, that is

$\Phi_{\mathrm{o}} \simeq 0 \Longrightarrow \epsilon=-\Phi_{\mathrm{o}} \simeq 0$.

In other words a small closure phase gives rise to an erroneously low value for the estimated error in the model. This wrong estimation of the error in the model propagates itself into a wrong estimation of the antenna error,

$E_{i}-E_{k}=\psi_{\mathrm{o}}-\psi_{\mathrm{m}}-\epsilon$,

which becomes $E_{i}-E_{k} \simeq \psi_{\mathrm{o}}$ since $\psi_{\mathrm{m}}$ and $\epsilon$ both vanish. As a result the corrected observed phase $\psi_{\mathrm{o}}^{\prime}$ is equal to

$\psi_{\mathrm{o}}^{\prime}=\psi_{\mathrm{o}}-\left(E_{i}-E_{k}\right) \simeq 0$.

In conclusion, small closure phase values mean that self calibration is unable to correct an erroneous initial point model. Only low estimations of $\epsilon$ are possible, i.e., the model is taken as a good approximation of source structure, and large antenna corrections are applied to the observed phase to force it to follow the model. The result is that an input model with small closure phase, characteristic of any symmetric source, will produce corrected phases $\psi_{\mathrm{o}}^{\prime}$ set to zero as well. This will produce the observed symmetrization: part of the flux density is displaced to make the source symmetric.

Let us now assume that the model phase determined by the set of clean components, produces, due to the use of 
proper CLEAN boxes, an $\epsilon$ smaller than before and more comparable to the small closure phase. In this case self calibration should converge toward the proper solution, i.e., each iteration should remove the bias of the preceding one. Why, then, does this not happen? In a linear process such as described in Eq. (10), the solution derived from a given iteration of self calibration (sc) is simply added to the solution from the previous iteration, that is

$$
\begin{aligned}
\left(E_{i}-E_{k}\right)_{\text {Loop }-\mathrm{A}}= & \left(E_{i}-E_{k}\right)_{\mathrm{sc}-1}+\left(E_{i}-E_{k}\right)_{\mathrm{sc}-2} \quad(12) \\
= & \left(E_{i}-E_{k}\right)_{\mathrm{sc}-1}+\left[\psi_{\mathrm{o}}-\left(E_{i}-E_{k}\right)_{\mathrm{sc}-1}\right. \\
& \left.-\psi_{\mathrm{cc}}+\left(\Phi_{\mathrm{o}}-\Phi_{\mathrm{cc}}\right)\right] \\
= & \psi_{\mathrm{o}}-\psi_{\mathrm{cc}}+\left(\Phi_{\mathrm{o}}-\Phi_{\mathrm{cc}}\right)
\end{aligned}
$$

where $\psi_{\mathrm{cc}}$ and $\Phi_{\mathrm{cc}}$ are the model phase and the model closure phase determined by the set of clean components. If the self calibration process were indeed linear, Eq. (12) would produce the same result as from a single iteration of Loop "B":

$\left(E_{i}-E_{k}\right)_{\text {Loop }-\mathrm{B}}=\psi_{\mathrm{o}}-\psi_{\mathrm{cc}}+\left(\Phi_{\mathrm{o}}-\Phi_{\mathrm{cc}}\right)$.

But we have shown empirically that in the case of Test-2

$\left(E_{i}-E_{k}\right)_{\text {Loop-A }} \neq\left(E_{i}-E_{k}\right)_{\text {Loop-B }}$.

This implies a non-linear equation for $\left(E_{i}-E_{k}\right)_{\mathrm{sc}}$.

As we will show in Sect. 4.2 self calibration is in fact a non linear process. This explains why Loop "B" works better than Loop "A". At each iteration Loop "B" discards the previous $\left(E_{i}-E_{k}\right)$ estimation and simply starts again, approaching the proper solution for the antenna corrections as $\psi_{\mathrm{m}} \rightarrow \psi_{\mathrm{t}}$. On the contrary Loop "A" accumulates previous, incorrect estimations and being unable to correct for them, biases the total antenna corrections even though $\psi_{\mathrm{m}} \rightarrow \psi_{\mathrm{t}}$. We have checked this surmise by setting CLEAN boxes to exclude the spurious features, forcing the CLEAN model to be the "true" one. Self calibration nevertheless determines corrections which still reproduce the artificial counter-jet.

However, if we accept that in general Loop "B" works better than Loop "A" since self calibration is non-linear, why does, in some cases, Loop "A" converge to the same solution as Loop "B"? There must be some linearizing quantity in Test-1 that is lacking in Test-2. We remind the reader that Test- 2 has the same $u-v$ coverage as Test-1, except for the single intermediate baseline.

\subsection{Condition for linearity}

In general as shown in Massi \& Comoretto (1990) the minimization of Eq. (1), dealing with the difference of two complex quantities, can be reduced to the minimization of the difference of their phases, only when three conditions are satisfied: only phase errors are present, good SNR in the data, and small errors in the model. When these three conditions are satisfied the problem reduces to the linear case.

The presence of large errors in the model violates the condition of linearity; we will see below quantitatively what "large" means. Let us consider in Eq. (1) only phase errors, i.e., that the gain terms $g_{i}=g_{k}=1$ and neglect the amplitude and weight terms. Then, minimizing Eq. (1) with respect to the phase error terms gives

$\sum_{i \neq k} \sin \left[\psi_{\mathrm{o}}-\psi_{\mathrm{m}}-\left(E_{i}-E_{k}\right)\right]=0$.

We see as the presence of a large error $\epsilon \equiv \psi_{\mathrm{o}}-\psi_{\mathrm{m}}-$ $\left(E_{i}-E_{k}\right)$ prevents the linearization of Eq. (15). Let us therefore expand the sine function including the first non linear term, as $\sin x=x-\frac{x^{3}}{3 !}$.

Equation (15) then becomes $\epsilon_{i k}+\left(\Phi_{\mathrm{o}}-\Phi_{\mathrm{m}}\right)-\sum_{i \neq k} \frac{\epsilon^{3}}{3 !}=0$, or

$\epsilon_{i k}=-\left(\Phi_{\mathrm{o}}-\Phi_{\mathrm{m}}\right)+\sum_{i \neq k} \frac{\epsilon^{3}}{3 !}$.

This implies that for the linear case, that is for Eq. (16) to be equal to the linear case expressed in Eq. (8), it must hold true that $\left|\left(\Phi_{\mathrm{o}}-\Phi_{\mathrm{m}}\right)\right| \gg \sum \frac{\epsilon^{3}}{3 !}$.

Assuming an initial point-like source, the condition for linearity is further reduced to

$\left|\Phi_{\mathrm{o}}\right| \gg \sum \frac{\epsilon^{3}}{3 !}$

In conclusion, self calibration may reduce to a linear equation only if the errors $\epsilon$ of the model, that is the difference between the phases of the model and the true ones are such that the combination of their cube is much smaller (at least one order of magnitude lower) than the observed closure phase.

Since the errors $\epsilon$ for a point-like model are exactly the phase of our simulated source from FAKE, we can illustrate this result.

Figure 9a shows the closure phase available in Test1 along with the combination of the errors. The closure phase is in this case more than one order of magnitude higher than the combination of the errors; these last appear in fact quite unsignificant in the plot. This implies that Eq. (17) is for Test-1 satisfied and as a consequence self calibration should reduce to the linear case, that is Eq. (12) should be satisfied as well. And in fact we have shown as Loop "A" give same results as Loop "B" for Test-1.

On the contrary we see in Fig. 9b that when the closure phase drops to a few degrees, as is the case for unbalanced triangles, the errors in the model, previous negligible, become a significant fraction of the closure phase. In other words the condition of Eq. (17) no longer applies and the non linear terms in self calibration cannot be neglected; we are therefore in the condition expressed in Eq. (14). A conclusion verified in Sect. 2 with test 2 which gives different results for Test-2A and Test-2B. 

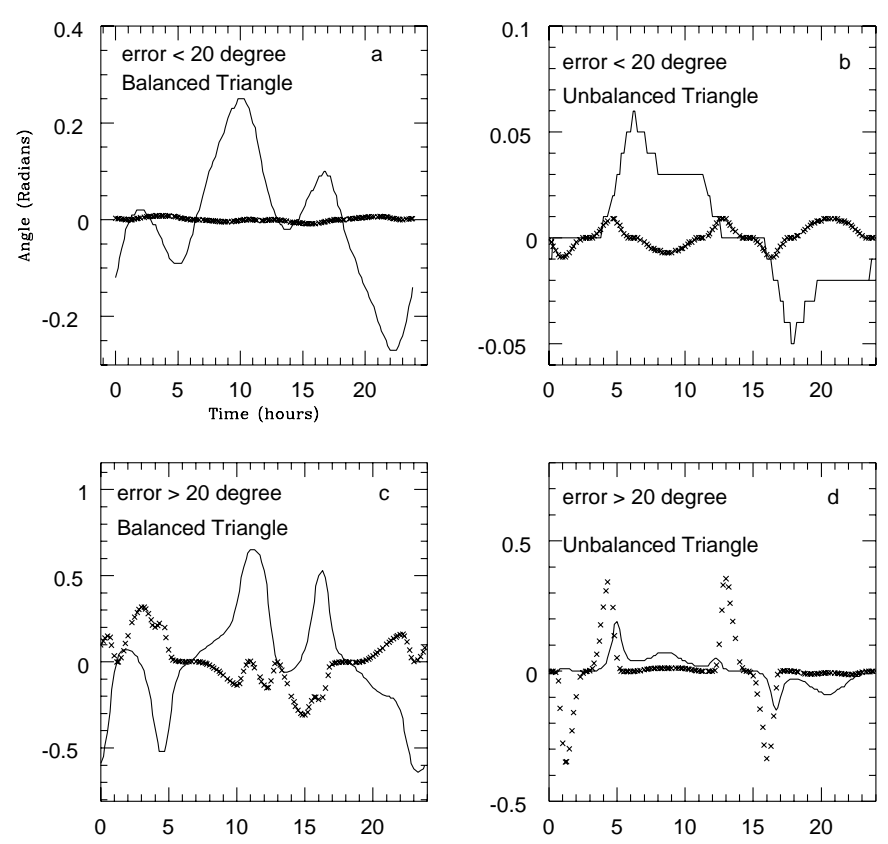

Fig. 9. Closure phase (continuous line) and non-linear error terms (crosses) in the self calibration solution (see Sects. 4 and 5)

Figure 9c illustrates that when the source has a complicated structure and a point source has been used as an initial model, the errors are so large to become an important fraction of the closure phase even for large closure phase values. In the case of Fig. 9d finally we show the worst combination of small closure phase values and large errors giving rise to a solution dominated by the non linear terms.

\section{Conclusion}

The hybrid mapping procedure may fail when the observed closure phases are only a few degrees while the individual baseline phases are large quantities. This happens when one telescope is far removed from the others of the network. In the resulting unbalanced triangles the two longest baselines become nearly parallel giving essentially identical phases but with opposite sign; their contributions therefore almost cancel in the closure equation. When the source is unresolved on the remaining, short baseline, the result is a closure phase of a few degrees.

Moreover, even in the presence of large closure phase values, the hybrid mapping procedure may fail when an oversimplified initial model is used to map a complicated source structure.
In both cases, low closure phase values or large errors associated with the model, hybrid mapping fails, generating artificial structures.

We demonstrate that the general precondition to avoid false structures in the map is that the errors (or more precisely their cube) of the model should be smaller than the observed closure phases. When this condition is satisfied the equation used in self calibration becomes linear.

It is evident that the small closure phase values associated with unbalanced triangles make this upper limit quite low and therefore easy to violate. When this condition is violated, the equation used in self calibration is non-linear and we can no longer assume that the antenna corrections are a sum of individual estimates obtainable by successive iterations. This procedure, which is normally followed in VLBI data processing, and is what we call in this paper Loop "A", fails in this case. The non-linear equation produces antenna corrections little modified by successive cycles of self calibration even if, as shown in this paper, new, better models are tried subsequently. In other words the wrong initial corrections, remaining nearly unmodified, corrupt irremediably the data.

In this case the correct procedure is simply to self calibrate again, adopting the model derived by CLEAN directly on the original data and not on the corrected ones biased by the previous wrong solution.

This is what we call Loop "B". At each iteration Loop "B" discards the previous accumulated corrections and starts to compute them again as a whole by using the new better model derived by CLEAN. When this is done at each iteration the use of progressive better models ensure the above given condition for linearity to be satisfied and therefore the convergence on the proper solution.

Acknowledgements. We would like to thank Guidetta Torricelli for her useful suggestions and Richard Porcas for his comments. We both acknowledge support for our research by the European Union, M.M. under contract FMGECT950012, S.A. under contract CHGECT920011.

\section{References}

Bååth, 1989, Very Long Baseline Interferometry. Techniques and Applications, Felli M. and Spencer R. (eds.). Kluwer Academic Publishers, NATO ASI Series C 283, 206

Linfield R.P., 1986, AJ 92, 21

Massi M., 1989, A\&A 208, 392

Massi M., Comoretto G., 1990, A\&A 228, 569

Schwab F.R., 1980, Proc. Soc. Photo-Opt. Inst. Eng. 231, 18

Walker R.C., 1986, Synthesis imaging, Perley R.A., Schwab F.R. \& Bridle A.H. (eds.). NRAO Workshop, p. 202 